\title{
Ionization Laser Calibration System for DUNE
}

Darcy A. Newmark (On behalf of DUNE Collaboration) | Los Alamos National Laboratory

\section{Overview of Deep Underground} Neutrino Experiment (DUNE)

- Next generation long-baseline neutrino experiment from Fermilab to SURF, South Dakota

- Far detector consists of four 10-kton liquid argon time projection chambers (LArTPCs)

- Construction will begin in 2024 and neutrino data taking expected in late 2020s

Primary Physics Goals

- Resolve neutrino mass hierarchy

- Measure $\delta_{\mathrm{cp}}$ (charge parity violating phase) in the neutrino sector - Search for supernovae events

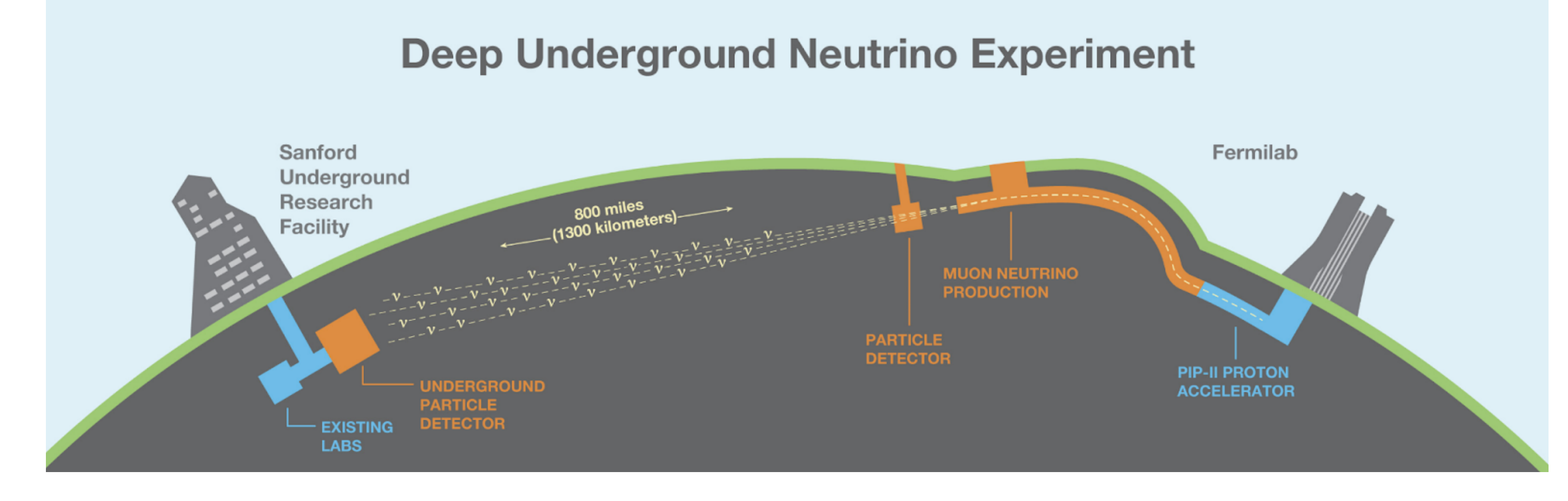

Figure 1. The DUNE experiment

\section{Calibrating the DUNE Far Detector}

- Understanding the response of the detector is crucial to achieve the energy scale and resolution necessary for the physics goals of DUNE

\section{Calibration Challenges}

- Huge size

- Highly segmented detector

- Hundreds of anode and cathode planes

- Cosmic ray muons at a lower rate due to deep underground location

Ionization laser (loLaser) system is one of the primary calibration systems planned for DUNE
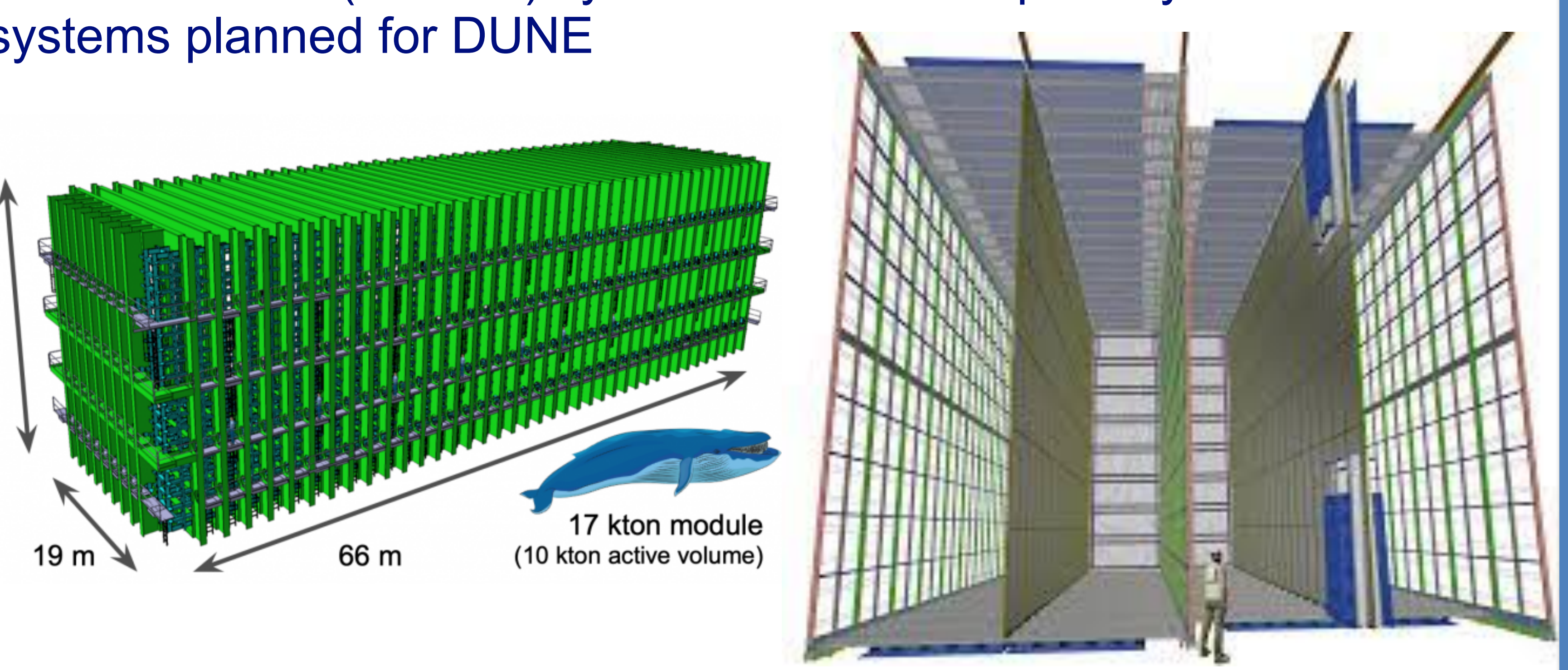

Figure 2. Single far detector LArTPC module (left) and

\section{Ionization Laser Calibration System} Create straight, well-defined ionizing

laser tracks in detector for calibration

- Provide fine grained measurements of detector parameters

- E.g. drift velocity, electric field distortions

Serve as diagnostic tool

- E.g. identify anode/cathode tilts etc.

Three main components

1. Class IV ionizing laser

2. Optical bench to direct a well

collimated, clean beam of $266 \mathrm{~nm}$ light

3. Periscope and feedthrough assembly to direct and rotate the beam into the Ar detector

On the order of 12 loLaser systems anticipated for a single detector module - Currently building two full loLaser

systems at LANL

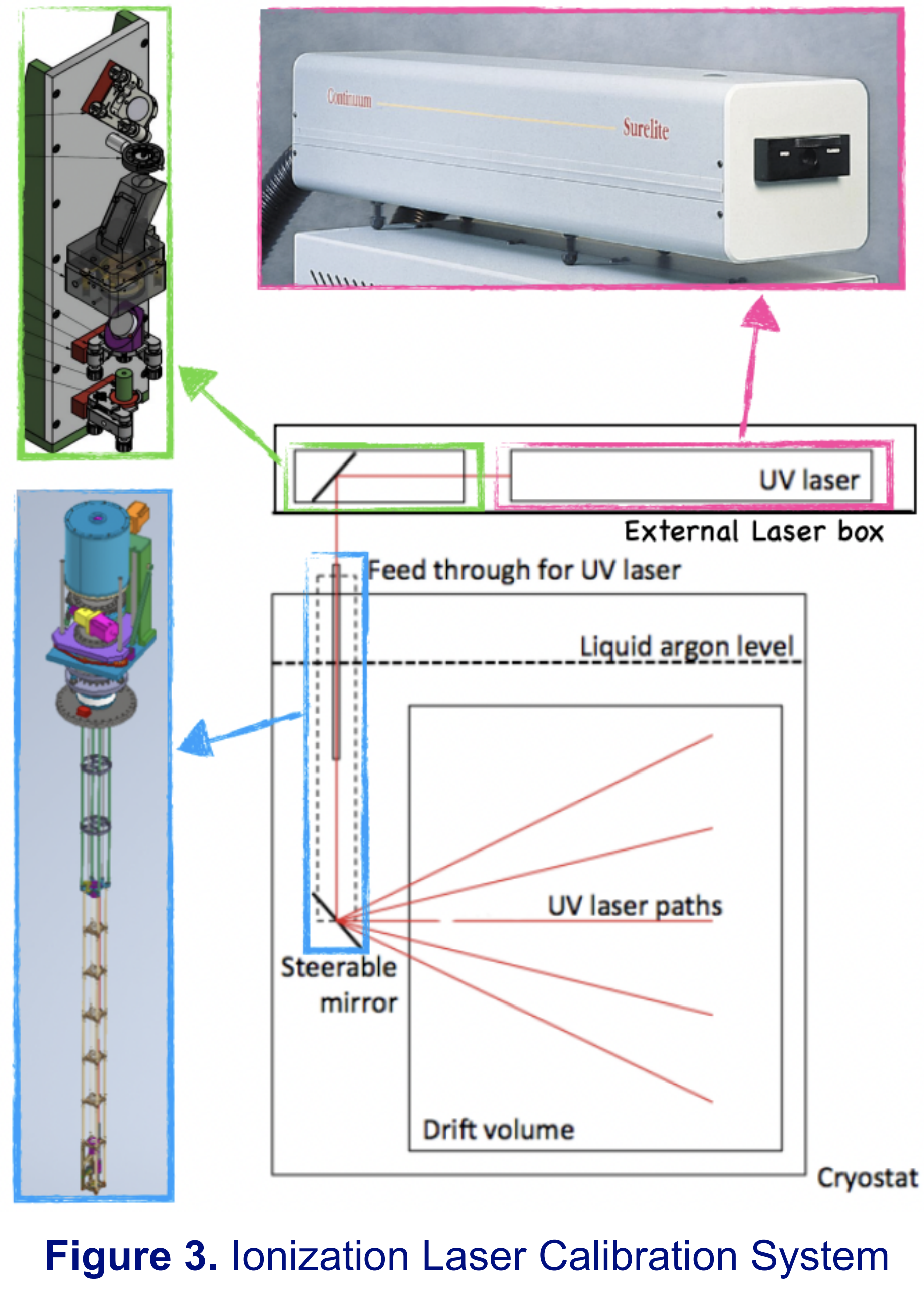

\section{Laser and Optical Bench}

\begin{tabular}{l|l}
\multicolumn{1}{|c|}{ Component } & \multicolumn{1}{c|}{ Purpose } \\
\hline $\begin{array}{l}\text { Surelite I10 } \\
\text { Nd:YAG class IV } \\
\text { Laser }\end{array}$ & $\begin{array}{l}\text { Emits } 1064 \mathrm{~nm}, 532 \\
\mathrm{~nm} \text {, and } 266 \mathrm{~nm} \text { light }\end{array}$ \\
\hline $\begin{array}{l}\text { Low power visible } \\
\text { laser }\end{array}$ & $\begin{array}{l}\text { For alignment } \\
\text { purposes }\end{array}$ \\
\hline
\end{tabular}

Beam splitter $\quad$ Separate $266 \mathrm{~nm}$ light

mirror from other

wavelengths

Dual band mirror Transfer both $266 \mathrm{~nm}$ and $532 \mathrm{~nm}$ light

Attenuator

Control laser energy

Iris

Reduce beam diameter
PNE DEEP UNDERGROUND

Los Alamos

\section{Optical Feedthrough and} Periscope

Two periscope designs to maximize coverage of the detector One for the central region (penetrates the TPC top field cage and includes a retraction device for safety)

- One for the end-wall region (send light from outside the field cage using a dual rotary motion)

- Periscope designs final, procurement and fabrication ongoing LANL and LIP

Periscope includes a number of seals to the cryostat which need to be tested for leak tightness $\left(10^{-6}-10^{-8} \mathrm{mbar}^{*} / \mathrm{s}\right)$

Helium leak testing of components (e.g. rotary stages, flange view ports) ongoing at LANL and LIP (see Fig. 5)

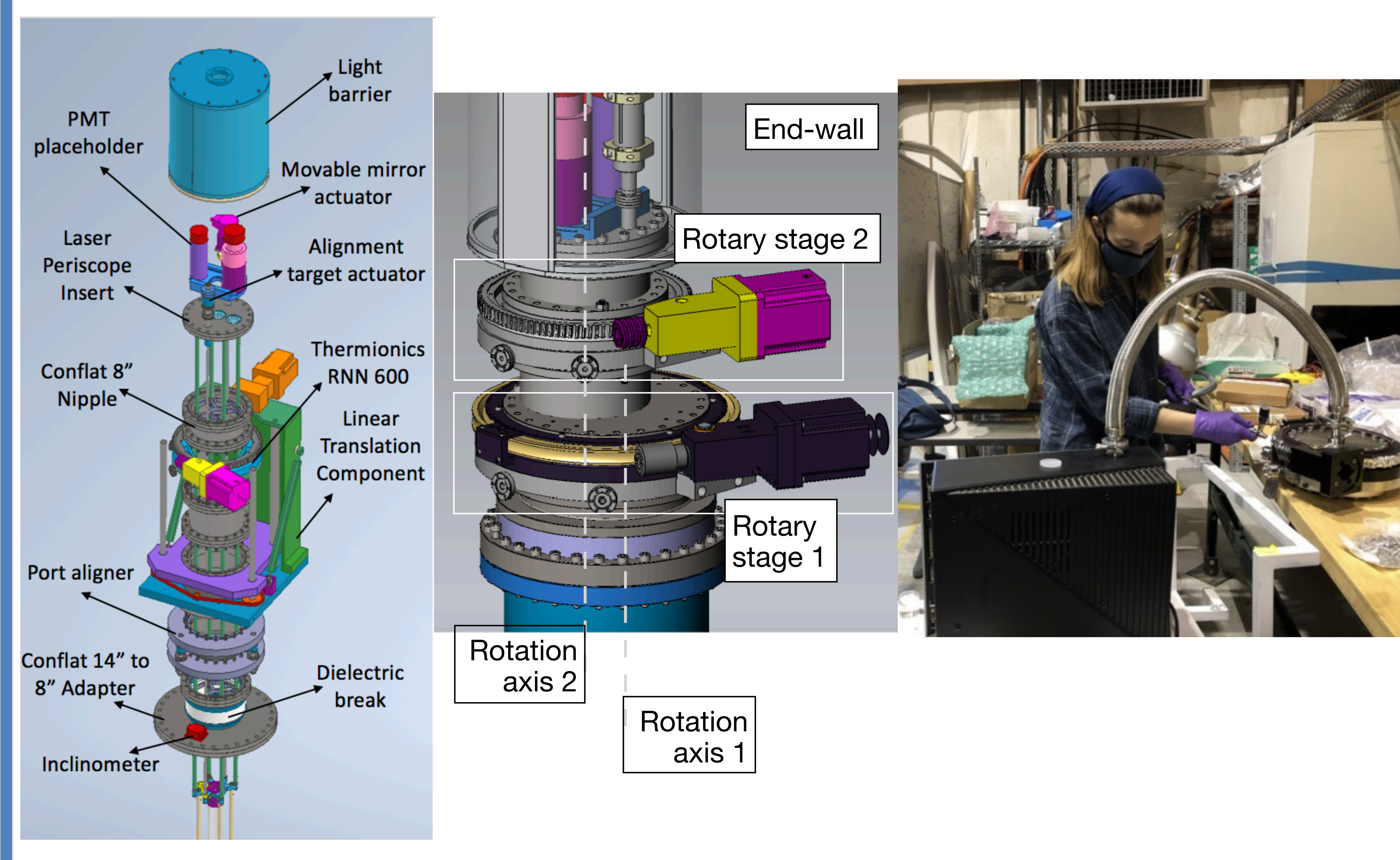

Figure 5. Top-field cage periscope design (left), end-wall periscope design (center) and Newmark performing leak test on RNN-600 rotary stage at LANL (right)

\section{Summary and Next Steps}

- Developing ionization laser calibration system to perform fine grained measurements of detector parameters and diagnose detector issues

- Finalizing optical setup to extract well collimated beam of $266 \mathrm{~nm}$ light

- Building 700 liter cryogenic test stand at LANL to test full ionization laser calibration system in LA

- Two full prototype laser systems will be installed in DUNE's 400 ton Prototype detector (ProtoDUNE) at CERN in early 2022. 